\title{
Adaptation to Suppression of Visual Information During Catching
}

\author{
Francesco Lacquaniti and Claudio Maioli \\ Istituto di Fisiologia dei Centri Nervosi, CNR, Milan, Italy
}

\begin{abstract}
We address the problem of whether and how adaptation to suppression of visual information occurs in catching behavior. To this end, subjects were provided with advance information about the height of fall and the mass of a ball and an auditory cue signaled the time of release. Adaptation did occur, as indicated by the unimpaired ability to catch the ball without vision; however, it involved a major reorganization of the muscle responses. The subjects were unable to produce anticipatory activity consistently, but preset the responses elicited by the impact. These responses were more complex and prolonged than those observed in the control experiments (with vision). In particular, medium- and long-latency responses were much larger, and the changes in elbow, wrist, and metacarpophalangeal angles following impact were more oscillatory than in the control. The general pattern of the EMG responses switched from that characteristic of catching with vision to that characteristic of catching without vision from the first trial of each experiment. However, the responses produced without vision were calibrated adaptively in the course of an experiment. In fact, the limb oscillations induced by the impact were significantly larger in the first trial than in the following trials. This seems to suggest that the parameters of the responses are adjusted based on an internal model of the dynamic interaction between the falling ball and the limb. This model is initially constructed from a priori knowledge on impact parameters and is subsequently updated on the basis of the kinesthetic and cutaneous information obtained during the first trial.
\end{abstract}

In the preceding paper, we showed that catching a falling ball in the presence of vision involves anticipatory and reflex coactivation of flexor and extensor muscles of the wrist and elbow joints (Lacquaniti and Maioli, 1989). We argued that these responses are centrally preset and, being precisely tuned to the physical parameters of ball impact, contribute significantly to limb stabilization.

Under normal conditions, the estimate of impact parameters and the production of an appropriate motor response rely on visual inputs, as well as on proprioceptive, exteroceptive, and possibly auditory inputs, all interacting within the framework defined by mental set and expectation. Thus, one may ask whether and how adaptation to suppression of visual information occurs in catching behavior, provided advance information about the height of fall and the mass of the ball is given to the subject and an auditory cue signals the time of release.

\footnotetext{
Received Feb. 10, 1988; revised May 17, 1988; accepted May 23, 1988.

Correspondence should be addressed to Dr. Francesco Lacquaniti, Istituto di Fisiologia dei Centri Nervosi, CNR, Via Mario Bianco 9, 20131 Milan, Italy.

Copyright @ 1989 Society for Neuroscience 0270-6474/89/010149-11\$02.00/0
}

A priori, the manner in which the system adapts to the suppression of a relevant sensory input might not be unique. One possibility is that the system has the capability of utilizing different sensory information to produce an invariant motor output. For example, this modality of adaptation to altered visual conditions occurs in the context of the postural control of the body during either free-fall (Greenwood and Hopkins, 1976; McKinley and Smith, 1983; Dyhre-Poulsen and Laursen, 1984) or anteroposterior translation (Berthoz et al., 1979). These studics consistently indicate that voluntary and reflex responses of limb muscles are not appreciably modified when vision is suppressed. Since these responses are greatly affected by sensory conflicts, such as those arising when the visual image is artificially stabilized (Nashner and Berthoz, 1978; Vidal et al., 1979), one can conclude that the information provided by each sensor can be weighed in a different and context-dependent manner (cf. Talbott and Brookhart, 1980; Nashner et al., 1982). Al the extreme case, sensory cues of different origin can effectively substitute for visual cues. Sensory substitution does also occur after labyrinthectomy. In fact, both visual inputs (Putkonen et al., 1977; Lacour et al., 1981; Maioli, 1988) and proprioceptive inputs from the neck (Dichgans et al., 1973) can substitute for the deficient vestibular inputs in the reflex control of limb, neck, or eye muscles.

A different possibility is that no such sensory substitution takes place, and adaptation to altered conditions involves, instead, a reorganization of the motor output. Motor substitution has been investigated to a lesser extent than sensory substitution as a potential mechanism of adaptation. It has recently been invoked to account for some types of eye movement adaptation to either vestibular deficits or conflicting visual-vestibular interactions (due to reversing prisms): Sequences of saccades can compensate for a nonfunctioning or inappropriate vestibuloocular reflex in the control of gaze (Berthoz, 1988; Melvill Jones et al., 1988). This is germane to the concept of motor equivalence, which is well established in the field of kinematic studies of multijoint coordination (see Abbs and Cole, 1987): different motor patterns can be utilized to attain the same goal.

Here we will show that the adaptation to visual suppression involves a major change in the motor strategy utilized for catching: All the burden of stabilizing limb posture is shifted onto muscle responses which are reactive to the impulsive perturbation. Functionally, the adapted behavior is a motor equivalent of the normal behavior, since both lead to an effective performance.

\section{Materials and Methods}

In a first series of 12 experiments, the set-up and procedures were similar to those described in the preceding paper. Briefly, the subjects were asked to catch a $0.4 \mathrm{~kg}$ ball that was dropped from one of 3 different heights $(0.4,0.8,1.2 \mathrm{~m})$. They were seated with their arm strapped to 
a goniometer, which measured elbow $(\theta)$ and wrist $(\Phi)$ angles. The goniometer was coupled to the shaft of a motor, thereby restricting limb motion proximal to the elbow (see Fig. 1 of Lacquaniti and Maioli, 1989).

In a second series of 4 experiments, limb motion was unconstrained. In this case, the subjects stood erect with their hand on the line of fall of the ball. The position of different limb segments was recorded by the ELITE system (Lacquaniti et al., 1987). To this end, a $50 \mathrm{~Hz}$ TV camera was placed orthogonally to the plane of limb movement (sagittal plane) and monitored small reflective markers attached to the skin overlying bony landmarks at the shoulder, elbow, wrist, fifth metacarpophalangeal (MCP) joint and little finger. The angles of flexion-extension at the shoulder $(\alpha)$, elbow $(\theta)$, wrist $(\Phi)$, and MCP joint $(\Psi)$ were computed trigonometrically from the $x, y$ coordinates of the limb markers.

EMG activities of biceps, triceps, flexor carpi radialis (FCR), flexor carpi ulnaris (FCU), extensor carpi radialis (ECR), and extensor carpi ulnaris (ECU) were recorded in all experiments. In 2 experiments, flexor pollicis longus (FPL) was also monitored.

Experimental protocol. In each experiment, 2 conditions were tested: vision permitted or excluded. In the latter case, subjects were asked to close their eyes throughout the duration of each trial. The ball was dropped at a random time $(1-4.5 \mathrm{sec})$ after a warning tone. A second tone was presented simultaneously to ball release, except in a low percentage of the trials $(<10 \%)$, randomly selected among those in which vision was excluded. In each experiment, blocks of at least 20 consecutive trials were run for any given height of fall both in the presence and in the absence of vision, the order being randomized across experiments. Note that subjects always knew the height of fall.

Data analysis. General processing of the data was as in the previous paper. In particular, all trials, except the first 3, of each series were ensemble averaged. Quantitative analysis was always performed on these ensemble averages, except for the cases mentioned in the text.

The EMG responses following impact tended to be fractionated in multiple components. We shall define as short-latency components those occurring within the $15-40$ mscc interval after impact, medium-latency components those within the $40-80 \mathrm{msec}$ interval, and long-latency components those within the 80-200 msec interval. Although such subdivision may be somewhat arbitrary, particularly when multiple peaks of activity merge, it helps to highlight the differences between the 2 visual conditions and to establish comparisons with other results in the literature. The mean amplitude of short-, medium-, and long-latency responses was computed over the predefined time windows and the baseline was then subtracted. The baseline was defined as the mean EMG activity over the $20 \mathrm{msec}$ interval centered on impact time.

As an estimate of the tendency of limb position to oscillate, we computed the sum $E$ of time-weighted absolute values of elbow and wrist angular velocities over the $800 \mathrm{msec}$ following impact:

$$
E=\sum t(|\dot{\Theta}|+|\dot{\Phi}|) \text {. }
$$

In order to study its variations in the course of an experiment, the parameter $E$ was computed in individual trials and converted to a standard score $E^{\prime}$ :

$$
E^{\prime}=(E-\bar{E}) / \mathrm{SD},
$$

where $\bar{E}$ and SD are the mean and standard deviation, respectively, of the results in the first 13 trials of each experimental series.

\section{Results}

When ball release was signaled by an acoustic tone but the subjects could not see the fall, their ability to catch the ball did not appreciably deteriorate. In fact, the trials in which subjects failed were very rare ( $2 \%$ of all trials). However, the kinematic and EMG patterns were drastically different from the control.

\section{EMG activities}

Figure 1 shows the ensemble averages obtained in 2 experiments in which the ball fell from $0.8 \mathrm{~m}$ in the presence $(A, C)$ or absence $(B, D)$ of vision. There are marked differences between these 2 conditions with regard to both anticipatory and reflex responses. Without vision, in fact, anticipatory responses are practically absent, but the responses following the impact are larger and more prolonged than in the control.
With vision, EMG anticipatory activity consistently developed over the last $100 \mathrm{msec}$ before impact in all muscles investigated and was accompanied by wrist and elbow flexion, in agreement with the previous results (Lacquaniti and Maioli, 1989). In contrast, when vision was prevented, modulation of the EMG activity prior to impact occurred only in sporadic single trials. Due to the large intertrial variability in both amplitude and timing of such anticipatory behavior, the modulation of the EMG baseline was generally limited in the ensemble averages (see Figs. $1, B, D ; 4 B$ ). In such averages, the mean EMG amplitude over the $50 \mathrm{msec}$ interval before impact was definitely smaller than in the control (pairwise Student's $t$ test, $p<0.01$ in each muscle; the mean ratio of values across muscles was $0.69 \pm 0.16 \mathrm{SEM}, n=6$ ). Correspondingly, the extent of limb movement prior to impact was greatly reduced.

As for the EMG responses following impact, we shall consider separately short-, medium-, and long-latency components (see Materials and Methods). Short-latency responses of flexor and extensor muscles of the elbow and wrist joints consisted in a brisk increase of activity developing over the $15-40 \mathrm{msec}$ interval after impact. Latency and mean amplitude of these responses did not differ significantly with eyes open or closed. The pattern of short-latency coactivation has been thoroughly described in the companion paper. By contrast, the short-latency responses of the FPL did differ in the 2 conditions, being much larger in the absence of vision (Fig. $1 D$ ) than in its presence (Fig. 1C).

Medium-latency responses (developing over the $40-80 \mathrm{msec}$ interval) were only inconsistently present with vision. (For instance, a peak of activity at about $70 \mathrm{msec}$ can be noted in the ECR and ECU in Fig. $1 A$.) Without vision, prominent mediumlatency responses were consistently present in all muscles from the first trial of each experimental series. In contrast to the pattern of short-latency coactivation, medium-latency responses were most often organized reciprocally over pairs of antagonist muscles. Thus, a peak of activity in the biceps roughly coincided with a trough in the triceps (Figs. $1 B ; 5, A, C$ ). Similarly, a sequence of increment and decrement of activity in wrist flexors coincided with opposite changes in the activity of wrist extensors (Figs. $1 B ; 4 B ; 5, A, C$ ). However, a more complex relationship between the patterns of activity in antagonists was present in some instances (e.g., FCU and ECU in Fig. 1D), while the cases of strict coactivation were rare (biceps and triceps in Fig. $4 B$ ).

Not only did medium-latency responses occur more consistently in the absence of vision than in its presence, they also had a much larger amplitude (pairwise Student's $t$ test, $p<$ 0.001 in each muscle). For instance, the mean amplitude of biceps response was on average 25 times larger than in the control ( $n=11$; in 5 other experiments with vision, such mean amplitude was negative, indicating a decrement of activity below the baseline).

Long-latency responses were generally absent in the experiments in which vision was allowed. (In fact, in $84 \%$ of the cases, the mean EMG amplitude over the $80-200 \mathrm{msec}$ interval was negative.) Without vision, instead, appreciable responses were generally observed in all muscles. The pattern of the long-latency responses was more variable among experiments than that of the medium-latency responses. It also tended to be reciprocally organized over antagonist muscles.

The histograms plotted in Figure 2 comprise the time bins of all experiments in which the EMG activity of elbow and wrist 

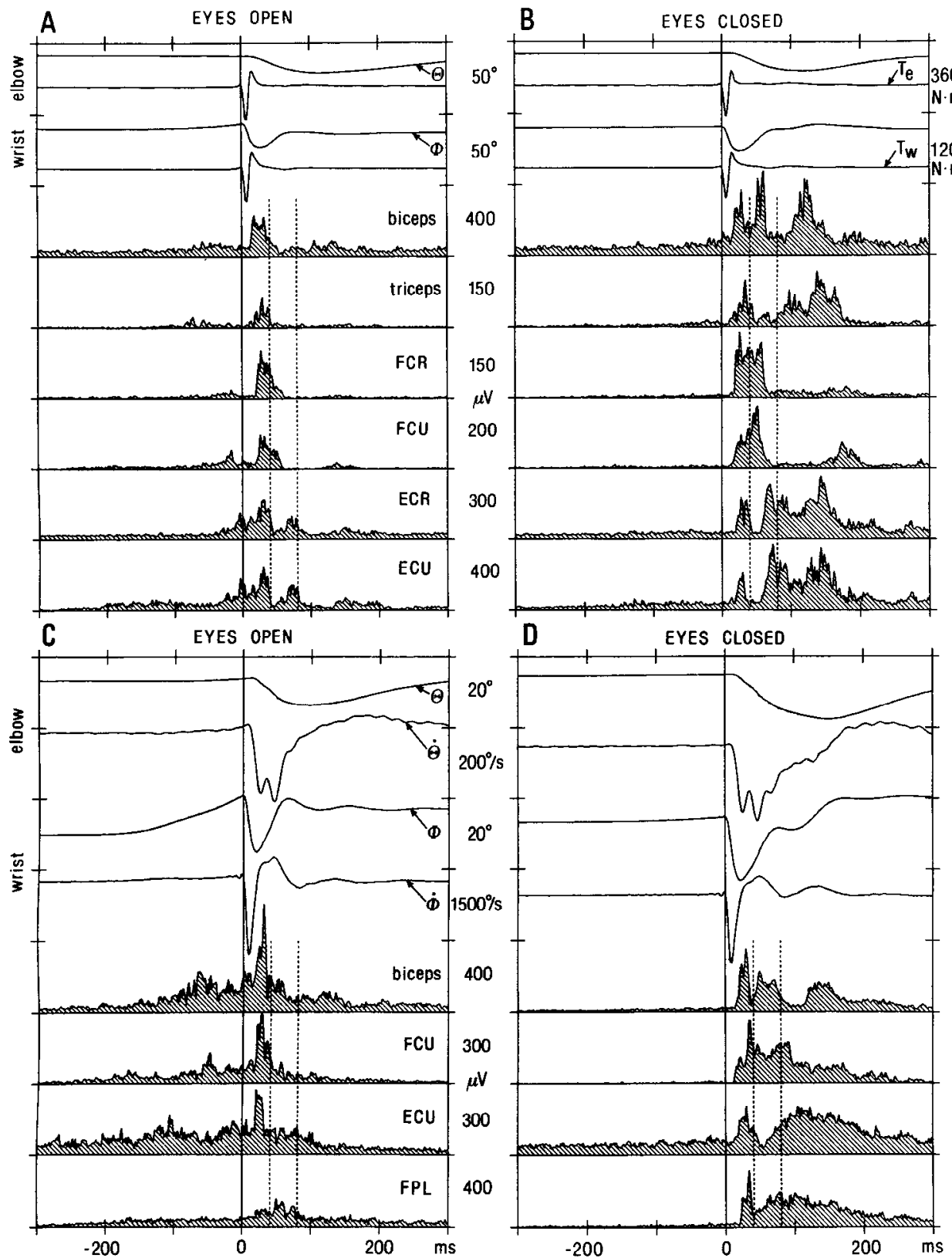

D

EYS CLOSED

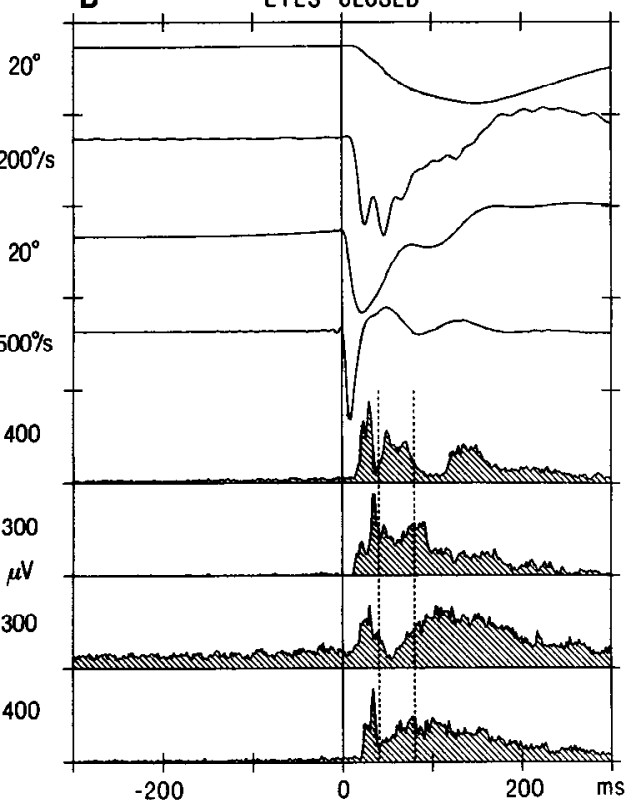

Figure 1. Catching with eyes open ( $A$, $C)$ or closed $(B, D)$. Ensemble averages obtained in 2 experiments (2 subjects, $A$ and $B$, and $C$ and $D$, respectively) in which a $0.4 \mathrm{~kg}$ ball was dropped from $0.8 \mathrm{~m}$ at $t \approx-400 \mathrm{msec}$ and hit the hand at $t=0 \mathrm{msec}$. $A$ and $B$, traces plotted from top to bottom correspond to elbow angle $(\theta)$ and torque $\left(T_{e}\right)$, wrist angle $(\Phi)$, and torque $\left(T_{x}\right)$, rectified EMG activity of biceps, triceps, flexor carpi radialis $(F C R)$, flexor carpi ulnaris $(F C U)$, extensor carpi radialis $(E C R)$, and extensor carpi ulnaris $(E C U)$. Calibration bars for $\theta$ and $\Phi$ are in the middle, those for $T_{e}$ and $T_{w}$ are on the right. $C$ and $D$, Traces from top to bottom correspond to elbow angle $(\theta)$ and angular velocity $(\dot{\Phi})$, wrist angle $(\Phi)$ and angular velocity $(\dot{\Phi})$, EMG activity of biceps, FCU, ECU, and flexor pollicis longus $(F P L)$. The 2 dotted vertical lines (at $t=40$ and $80 \mathrm{msec}$ after impact) delimit the time windows for the short-, medium-, and long-latency responses to impact. muscles after impact was significantly different $(p<0.05)$ from the basclinc. These histograms generalize the previous observations on the different duration of the responses according to the visual conditions. In the large majority of cases, when vision was prevented, EMG activity was significantly greater than the baseline over a much more prolonged time interval than it was in the presence of vision. (This statistical analysis is not apt, however, to reveal the patterns of reciprocal activation that are associated with EMG changes above the baseline.)

\section{Elbow and wrist kinematics}

Limb kinematics following ball impact also varied substantially according to whether vision was present or absent, namely, the changes in elbow and wrist angles were more oscillatory in the latter case (see Fig. 1). However, such differences were most pronounced about $50-100 \mathrm{msec}$ after impact. Thus, pairwise Student's $t$ tests revealed no significant differences between the
2 conditions in the peak values of the elbow and wrist torques and wrist angular velocities in extension, all occurring within the first $20 \mathrm{msec}$. In contrast, the peak angular position and velocity in extension at the elbow were significantly larger without vision than with vision. For instance, peak angular position at the elbow was $1.30 \pm 0.30(n=16)$ larger in the former case than in the latter.

The phase plane representation of elbow and wrist kinematics provided in Figure 3 best captures the differences between the 2 conditions. The angular velocity at the elbow is plotted versus elbow angular position in the top row of Figure 3, while wrist angular velocity and position are plotted in the bottom row. In each panel, the kinematic changes are plotted beginning with impact time and progress in the clockwise direction. Thick and thin traces correspond to the results obtained with eyes open or closed, respectively. Each column presents the results from a different experiment. 
Figure 2. Time histograms of the EMG responses evoked by the impact that were significantly different from the baseline. The time bins $(2 \mathrm{msec})$ in which the EMG activity of the indicated muscles was significantly $(p<0.05)$ above (black) or below (white) the prereflex baseline are plotted for all ensemble averages $(n-16) . t=0$ corresponds to the time of impact. Note that, in the large majority of cases, when vision was prevented (right) the EMG activity was significantly greater than the baseline over a much more prolonged time interval than it was in the presence of vision (left).
EYES OPEN
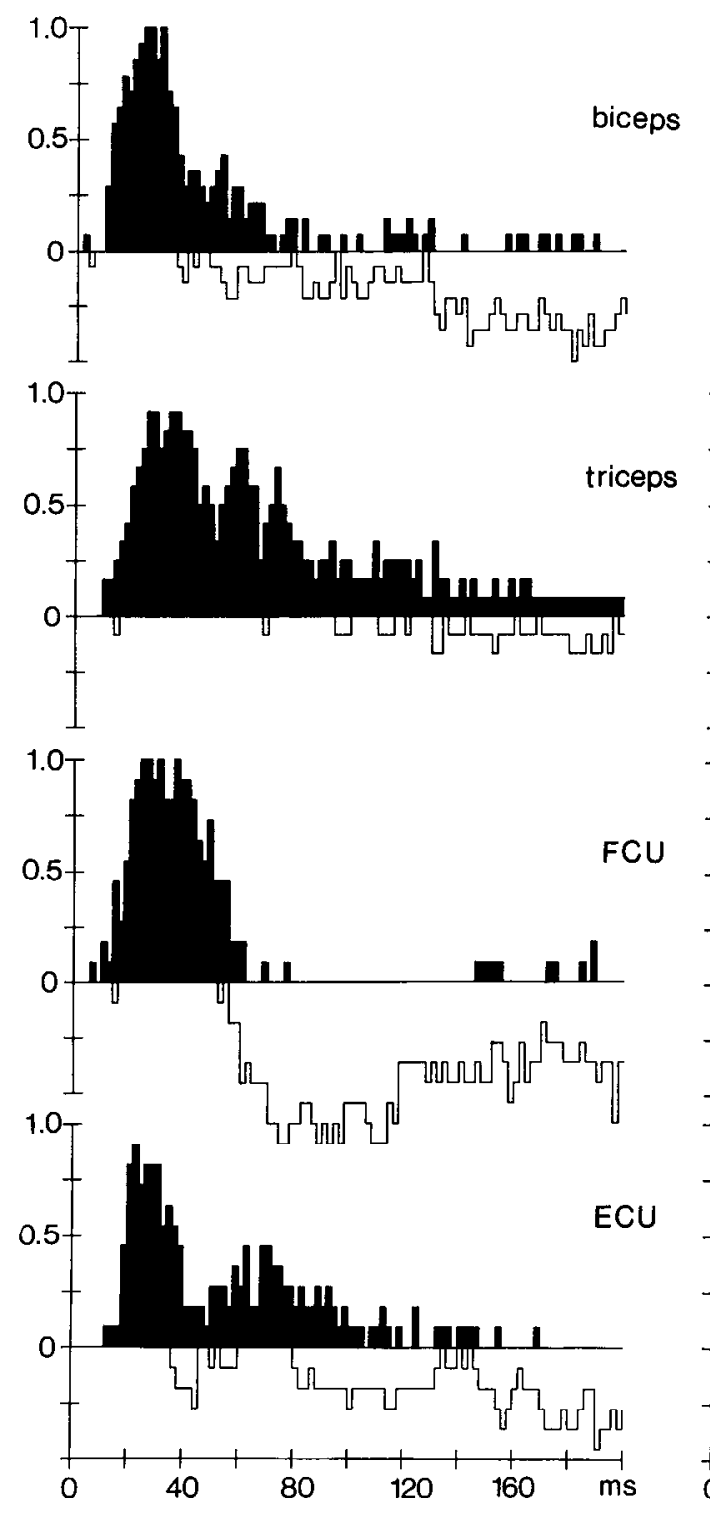

EYES CLOSED
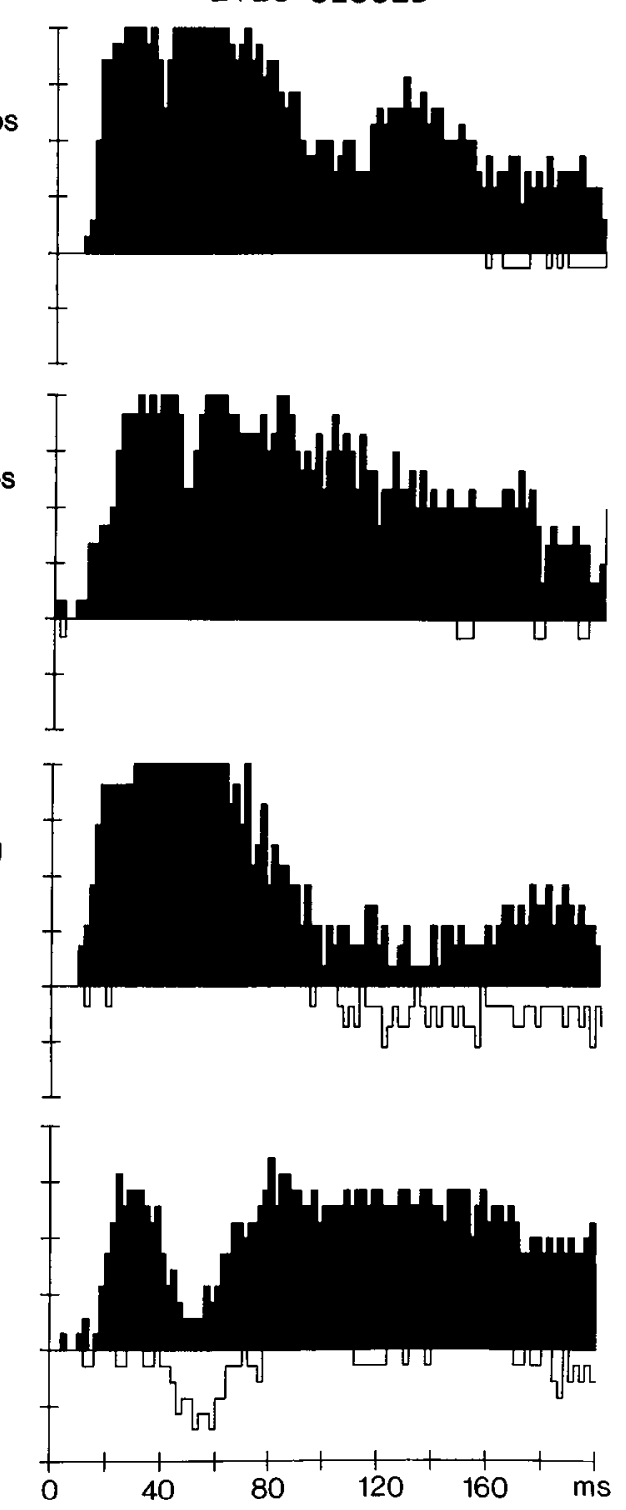

With vision, the phase plane trajectories tend to spiral towards a point of stable equilibrium which corresponds to the steadystate value of angular position and zero velocity of the elbow and wrist. Elbow trajectories obtained in the absence of vision have roughly the same shape as those obtained with vision but sweep over a larger area of the phase plane, due to the larger excursions in angular velocity and position. In contrast, the shape of wrist trajectories is clearly different in the 2 cases. The trajectories obtained in the absence of vision are characterized by a cuspidal singularity (indicated by the vertical arrowheads in Fig. 3). This corresponds to a point where movement transicntly stops and then resumes in the same direction as before. The timing of this point varies little among experiments; on average, it occurs at $91 \pm 22 \mathrm{msec}(n=16)$ after impact (see also Fig. $1, B, D$ )

As a quantitative estimate of the tendency of limb position to oscillate, we computed the sum $E$ of time-weighted deviations in elbow and wrist angular velocities (equation 1 in Materials and Methods). In the experiments performed in the absence of vision $E$ was $1.23 \pm 0.22$ greater than in the control.

\section{Grasping}

Lack of visual information involves not only a modification of the pattern of postural stabilization of elbow and wrist angular positions but also a modification of grasping. This is documented by the results presented in Figure 4.

In this experiment unconstrained limb motion was monitored by the ELITE system (see Materials and Methods). The angles of flexion-extension at the shoulder $(\alpha)$, elbow $(\Theta)$, wrist $(\Phi)$, and MCP joint $(\Psi)$ were then derived (see inset in Fig. $4 A$ ). The first observation to be made is that both kinematics and EMG activities at the elbow and wrist are similar to those previously described for the experiments in which limb motion proximal to the elbow was restricted. When the upper arm was free to move, backward extension at the shoulder, along with elbow and wrist extensions, was produced by the impact.

As for the changes in MCP angle, they were very different with or without vision. With vision (Fig. $4 A$ ), the subjects began to flex their fingers before impact to close the hand around the incoming ball. Finger flexion was accelerated by the impact of 

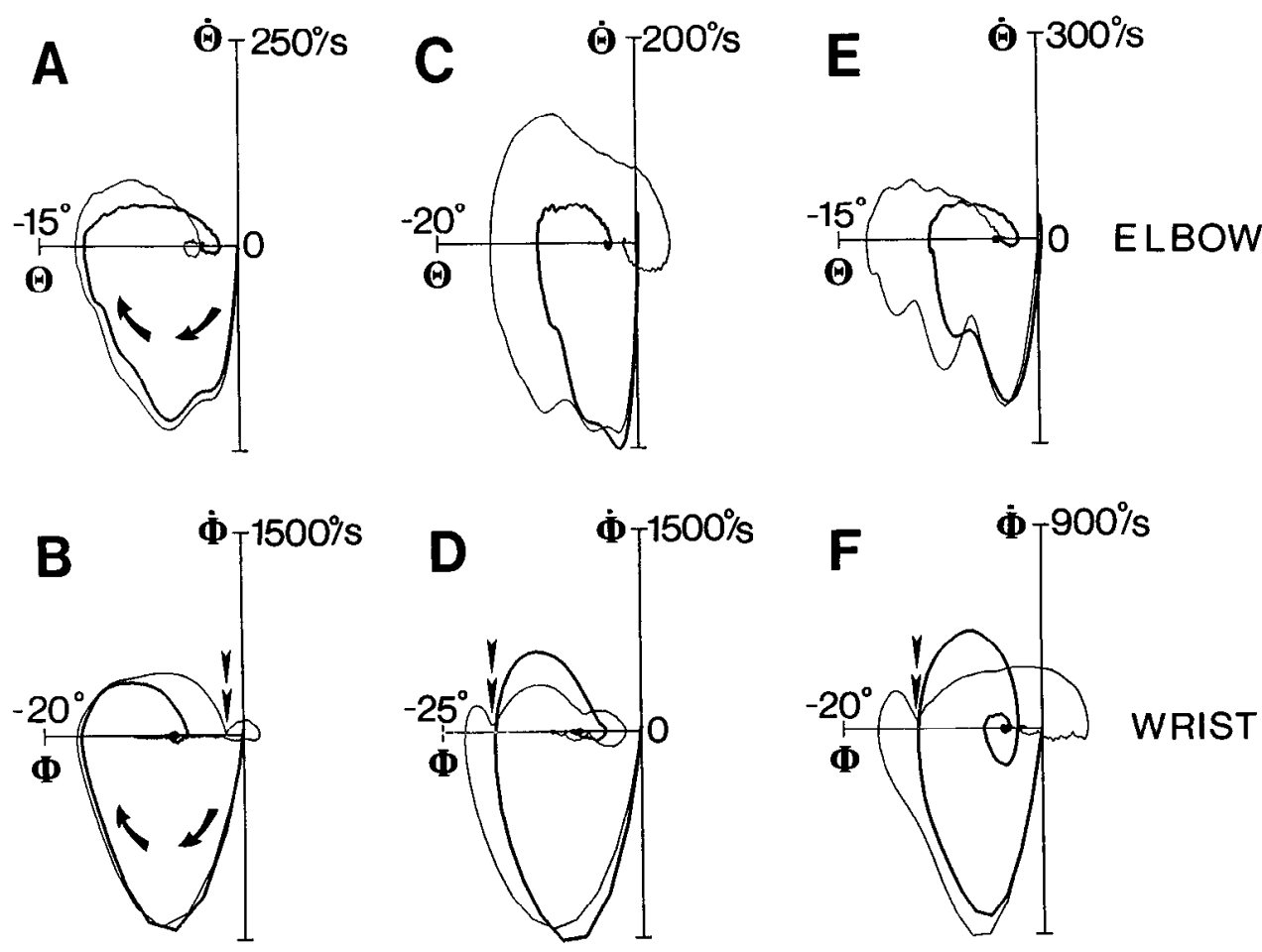

Figure 3. Phase plane representation of the kinematic changes following impact. Eibow angular velocity is plotted versus elbow angular position in the $t o p$ row, while wrist angular velocity is plotted versus wrist angular position in the bottom row. In each panel, the kinematic changes are plotted beginning with impact time and progress in the clockwise direction. Thick and thin traces correspond to the results obtained with eyes open or closed, respectively. Each column presents the results from a different subject and experiment (the ball fell from $0.8 \mathrm{~m}$ in $A$, and from $1.2 \mathrm{~m}$ in $B$ and $C$ ). the ball on the distal part of metacarpus, due to the dynamic coupling between finger motion and metacarpus motion. Grasping was then completed within the first $100 \mathrm{msec}$ after impact. By contrast, in the absence of visual information (Fig. 4B), anticipatory motion was reduced or absent. Furthermore, the initial flexion of the fingers due to impact was soon interrupted by an extension phase and resumed only $60-80 \mathrm{msec}$ after impact. Thus, grasping was completed $50-100 \mathrm{msec}$ later than in the presence of vision. It should be noted that the time at which finger motion reversed from extension to flexion corresponds roughly to the times at which wrist motion is transiently halted.

\section{Effect of surprise}

It could be argued that in the absence of visual information subjects are unable to preset their muscle responses and behave passively in the face of the applied perturbation. This hypothesis specifically predicts that the described reflex responses would be the same whether or not the subjects were alerted of the fall of the ball.

To test this hypothesis, in a few trials $(<10 \%)$ randomly interspersed with control trials in which vision was prevented, subjects were caught by surprise: After a random time interval from the first warning tone, the ball was released without the second tone. Figure 5 shows the results obtained in 2 such experiments. In the first (Fig. 5, $A, B$ ), movement of the upper arm was restricted and elbow and wrist angular motions were monitored goniometrically. In the second (Fig. 5, $C, D$ ), unconstrained limb motion was monitored by the ELITE (as in Fig. 4). Panels $A$ and $C$ show ensemble averages of the control trials in which the second tone was presented simultaneously with ball release; panels $B$ and $D$ show the averages of the trials in which this tone was suppressed.

Both the EMG responses and the kinematics following impact are clearly different according to whether or not the subject is alerted to the release of the ball. When the tone was suppressed, the mean amplitude of the short-latency reflex responses was significantly smaller than in the control (pairwise Student's $t$ test, $p<0.05$ in each muscle; the mean ratio of values was 0.61 $\pm 0.39, n=72$ ). Note that this difference reflects a real change in the gain of the responses, since the peak values of elbow and wrist torques (and angular velocities) did not differ significantly from the control values, nor did the prereflex EMG baseline (except in ECR and ECU, where it was smaller). Although shortlatency reflex coactivation of antagonist muscles did occur even when subjects were not alerted, the ratio between the amplitude of the responses in pairs of antagonist muscles was appreciably different from control, since the responses of extensor muscles (triceps, ECR, and ECU) were depressed to a greater extent than those of flexor muscles (biceps, FCR, and FCU). The mean amplitude of short-latency responses in extensor muscles was $0.46 \pm 0.53$ of the corresponding value in the antagonist muscle compared with the control ratio of $0.86 \pm 0.92$.

Both time course and amplitude of the EMG responses at latencies longer than $40 \mathrm{msec}$ differed substantially from those of the control responses. However, a direct comparison between these responses cannot be made because of the very large discrepancies in the kinematics after that time. In fact, the oscillations of the angular position of elbow and wrist joints were much larger and more prolonged in time than those observed in the control. Oscillatory behavior was quantified by computing the sum of time-weighted deviations in angular velocities. In the averages involving unexpected falls, this parameter was 1.81 \pm 0.62 greater than control averages. Finally, grasping of the ball was not very effective because of the delay in the flexion of the fingers (cf. Fig. 5, C, D), and in several instances subjects failed to catch the ball.

One can therefore conclude that, in the absence of visual information, the auditory cue on the timing of the ball's impact is indeed used to preset reflex responses that are adequate to sustain an effective catching. 


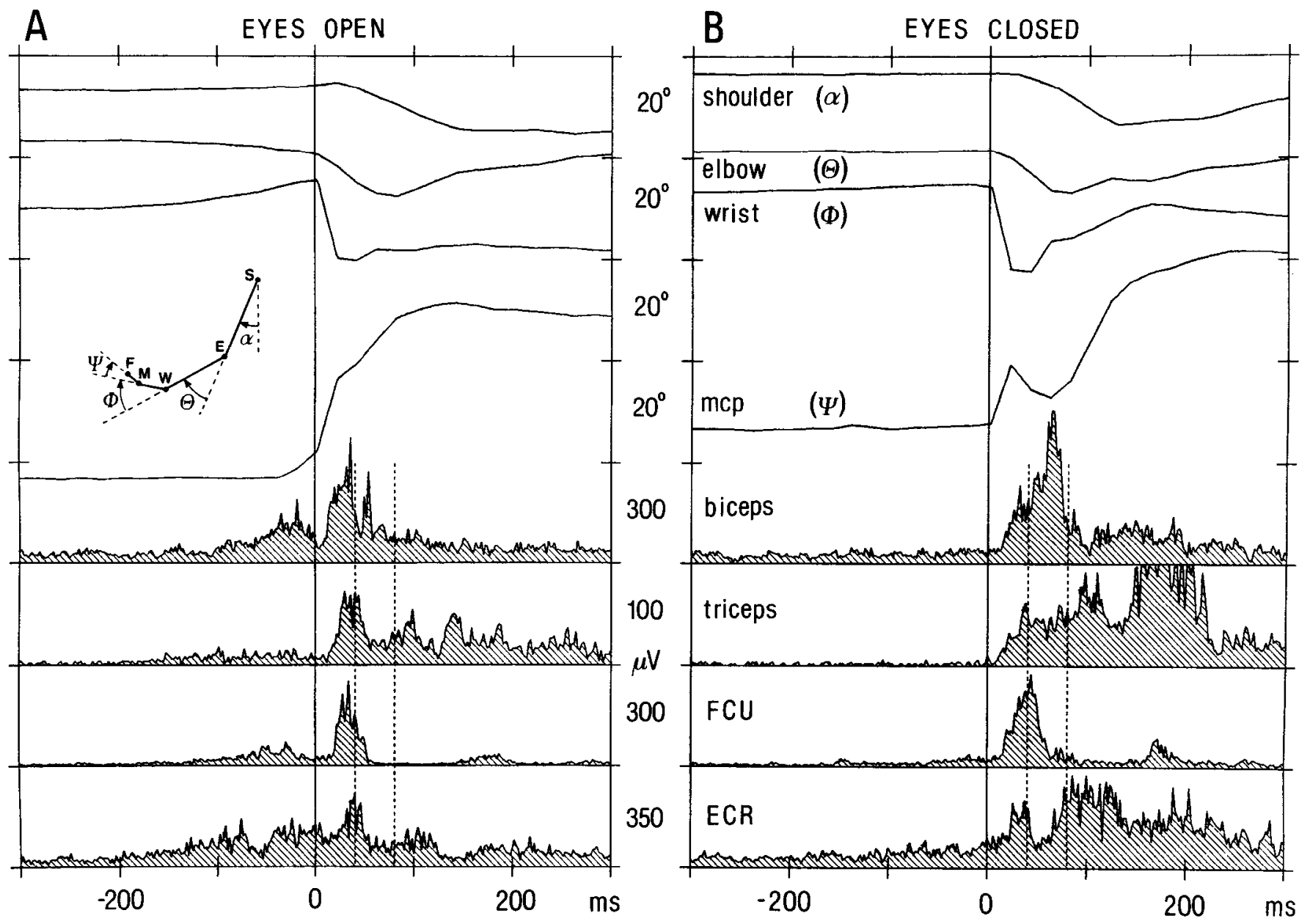

Figure 4. Ensemble averages from an experiment in which unconstrained limb motion was monitored by the ELITE system. A $50 \mathrm{~Hz}$ TV camera was placed orthogonal to the sagittal plane and monitored markers attached to the shoulder, elbow, wrist, fifth metacarpophalangeal $(M C P)$ joint, and little finger. The angles of flexion-extension at the shoulder $(\alpha)$, clbow $(\Theta)$, wrist $(\Phi)$ and MCP joint $(\Psi)$ were computed trigonometrically from the $x, y$ coordinates of the limb markers (inset in $A$ ). For illustrative purposes only, the data were not filtered. In this experiment, the ball was dropped from $0.8 \mathrm{~m}$ at $t \approx-400 \mathrm{msec}$.

\section{Adaptive calibration of the responses}

The evidence provided for the presetting of catching behavior raises the following question: Are the responses preset to a fixed value from the first trial of each experimental series, or are they instead modified adaptively in the course of an experiment? It can be recalled that the results discussed so far pertain to ensemble averages that comprise the fourth and following trials in any given series. We shall now compare the initial trials with the stationary ensembles.

In Figure 6, the changes in elbow and wrist angular positions following the impact are plotted for 10 different experiments performed in the absence of vision. The mean ( $\pm 1 \mathrm{SD}$ ) of the angular changes of the ensemble averages is plotted within the hatched pattern. Each ensemble comprises 10 consecutive trials beginning with the 4th of the series; the $1 \mathrm{st}$ trial is plotted individually.

In contrast to the appreciable variability of limb kinematics among experiments, the variability within each ensemble average is limited, indicating stationarity of the responses. However, the results of the first trials depart significantly from the averages. Limb kinematics in such trials is markedly more oscillatory than the average. ${ }^{1}$ As before, oscillatory behavior was quantified by computing the sum $E$ of time-weighted deviations in angular velocities. On average, in the first trials of all experiments this parameter was $1.64 \pm 0.58$ greater than in the ensemble averages.

In order to investigate the time course of the changes in oscillatory behavior, the parameter $E$ was computed in the first 13 trials of each experiment, and the corresponding standard score derived ( $E^{\prime}$ in equation 2; see Materials and Methods). The values of such standard scores have been averaged across all experiments and are plotted in Figure 7. Only the results corresponding to the first trials differ significantly from the mean values. ${ }^{2}$ Thus, adaptive calibration of the responses seems to take place in the space of one trial.

\footnotetext{
1 This was so independent of whether the series of trials with eyes closed was run before or after the corresponding series with eyes open at the same height of fall.

2 The statistical analysis performed on several EMG parameters (prereflex haseline, mean amplitude of the short-, medium-, and long-latency responses) yielded no consistent trends among experiments.
} 

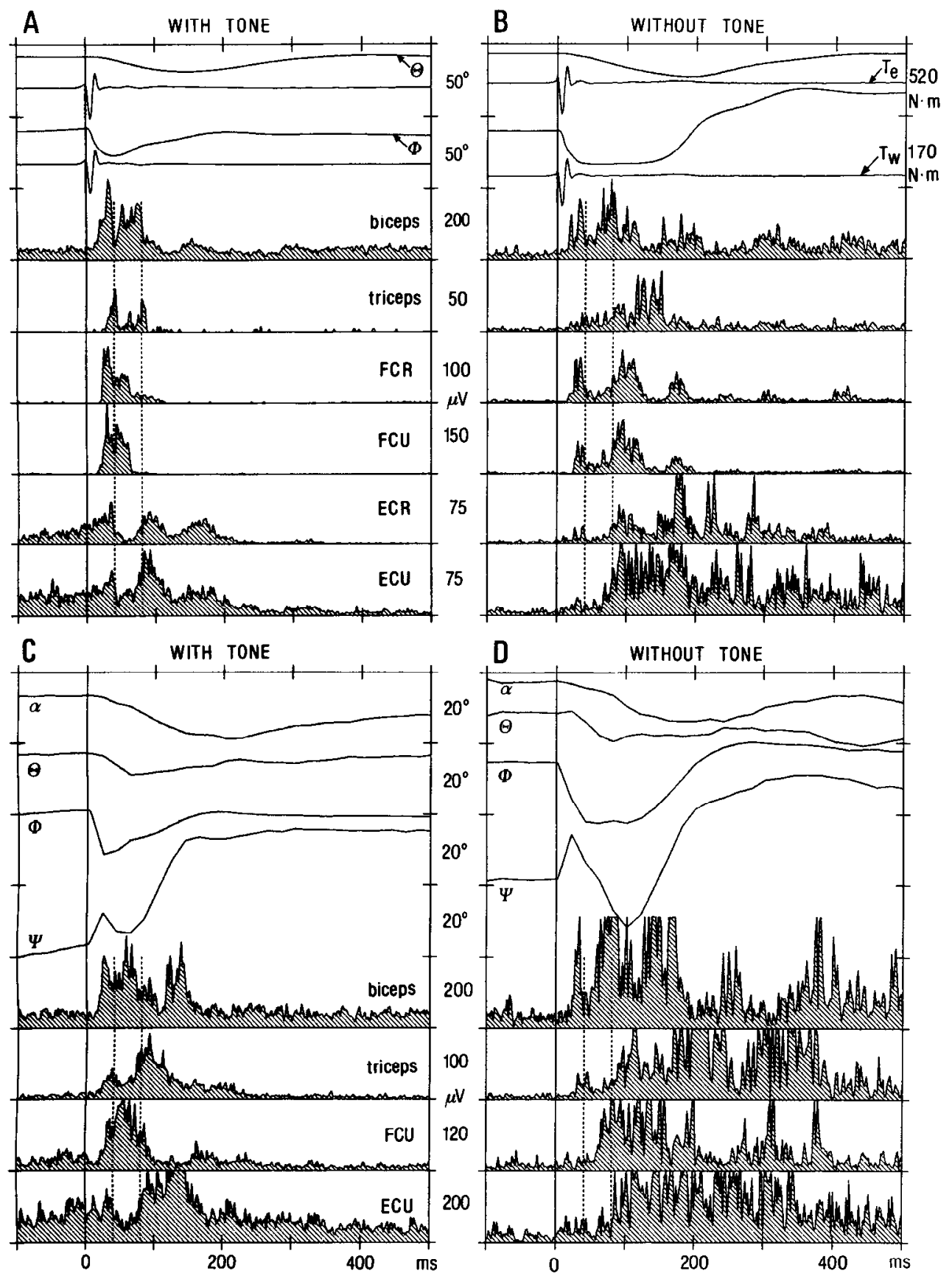

Figure 5. Effect of surprise. Ensemble averages from 2 experiments: in the first $(A, B)$, movement of the upper arm was restricted and elbow and wrist angular motions were monitored goniometrically; in the second $(C, D)$, unconstrained limb motion was monitored by the ElrTe (as in Fig. 4). In all cases, the ball was dropped from $0.8 \mathrm{~m}$ and vision was prevented. $A$ and $C$, The ensemble averages of the control trials $(n=45)$ in which an acoustic tone was presented simultaneously with ball release. $B$ and $D$, The averages of the trials $(n=4)$ in which this tone was unexpectedly suppressed. Such latter trials were randomly interspersed with the control trials. In $A$ and $B$, the traces from top to bottom correspond to elbow angle $(\Theta)$ and torque $\left(T_{e}\right)$, wrist angle $(\Phi)$, and torque $\left(T_{\text {,. }}\right)$, biceps, triceps, FCR, FCU, ECR, and ECU. In $C$ and $D$, the traces correspond to shoulder $(\alpha)$, elbow $(\Theta)$, wrist $(\Phi)$, and $M C P(\Psi)$ angles, biceps, triceps, FCU, and ECU.

\section{Discussion}

Although visual information plays a fundamental role in the control of catching behavior (Sharp and Whiting, 1974), adaptation to visual suppression does occur, albeit with a major reorganization of the muscle responses. With vision, the bulk of the modulation of muscle activity was concentrated around impact time. Anticipatory and reflex responses of brief duration resulted in a fast damping of the limb oscillations following impact and completion of grasping within a short time. In contrast, when the subjects were alerted of ball release by an auditory cue but vision was prevented, they were unable to produce anticipatory activity consistently. Nevertheless, motor preparation, involving a preset of the responses elicited by the impact, did occur even in these cases. These responses were more com- plex and prolonged than those observed in the experiments with vision. Correspondingly, the changes in elbow, wrist, and MCP angles following impact were also more oscillatory than in the control. Nonetheless, the overall performance was adequate for catching under our experimental conditions, as demonstrated by the very rare failures.

\section{Anticipatory responses}

The anticipatory EMG activity we described is reminiscent of the coactivation pattern beginning at a fixed interval of time prior to landing from a fall or jump (Greenwood and Hopkins, 1976; McKinley and Smith, 1983; Dyhre-Poulsen and Laursen, 1984). However, while the former is contingent upon vision, the latter is not. In fact, other sensory cues (most likely of vestibular origin), along with planning, can effectively substitute 

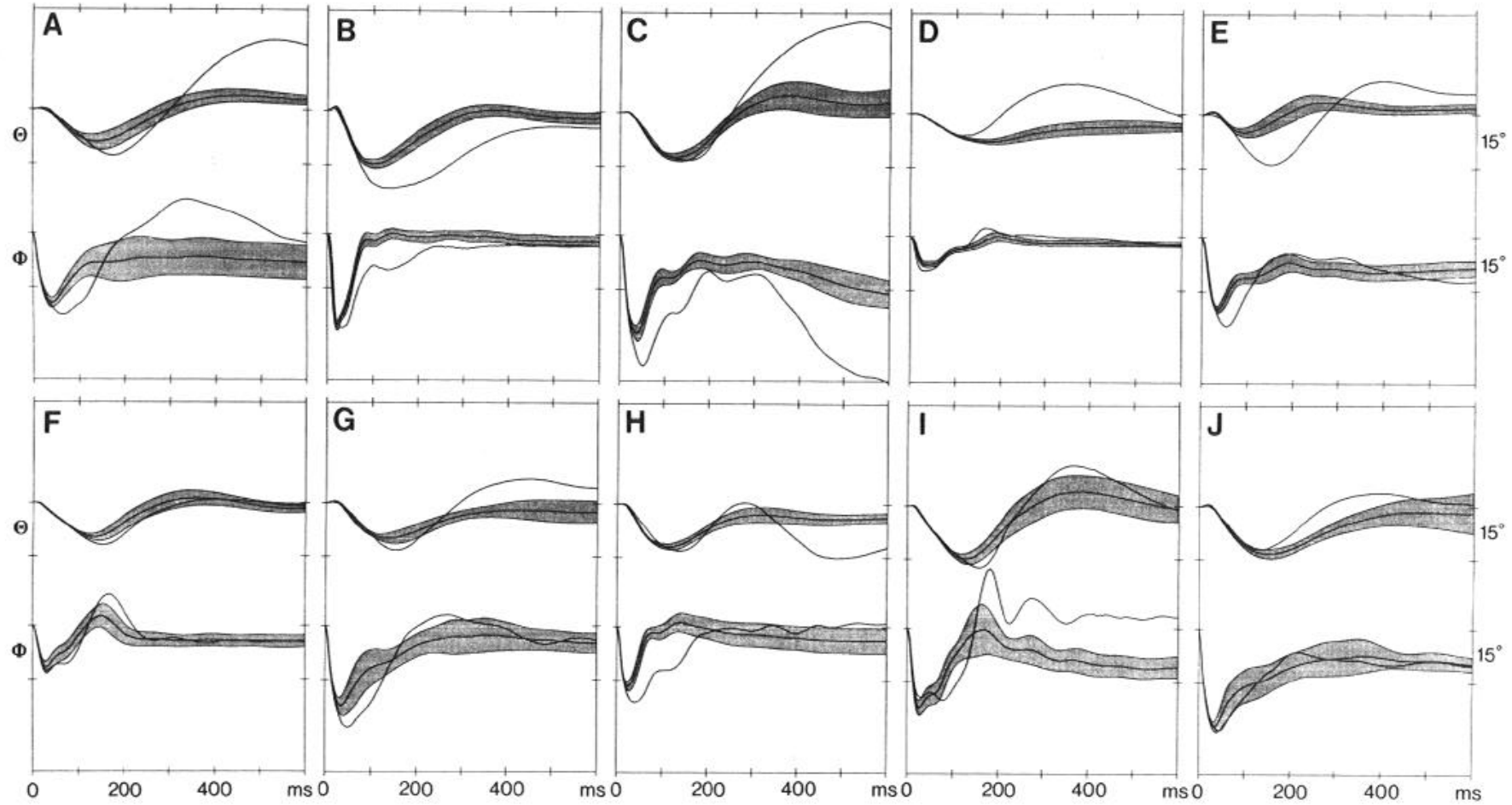

Figure 6. Adaptive calibration of the responses. The changes in elbow $(\Theta)$ and wrist $(\Phi)$ angles following the impact are plotted for 10 different experiments performed in the absence of vision. The mean $( \pm 1 \mathrm{SD})$ of the angular changes of the ensemble averages is plotted within the hatched pattern. Each ensemble comprises 10 consecutive trials beginning with the 4 th of the series; the 1 st trial is plotted individually in all cases. Note that limb kinematics in such 1st trials is markedly more oscillatory than in the averages.

for visual cues to elicit anticipatory behavior during landing, provided the falling height is known beforehand. The auditory cue on ball release supplied no equivalent substitution during catching. Perceptually, subjects appeared to be able to reach an estimate of the expected duration of fall even without vision, since they could detect the sporadic cases of inaccurate timing

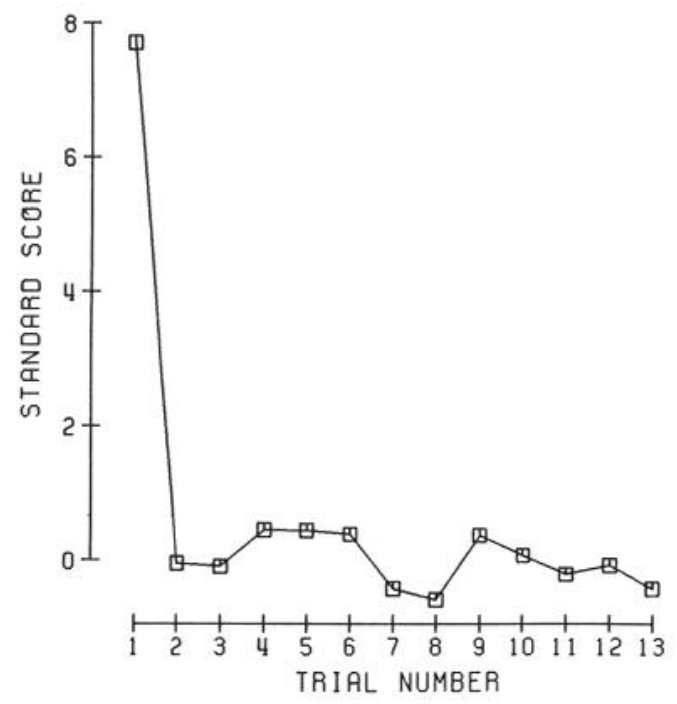

Figure 7. Time course of the adaptive changes. The sum $E$ of timeweighted absolute values of elbow and wrist angular velocities over the $800 \mathrm{msec}$ following impact was computed in individual trials and converted to a standard score $E^{\prime}: E^{\prime}=(E-\bar{E}) / \mathrm{SD}$, where $\bar{E}$ and SD are the mean and standard deviation, respectively, of the results in the first 13 trials of each experimental series. The values of such standard scores have been averaged over all experiments $(n=16)$. of the auditory cue (in such cases the tone led ball release by a few tens of milliseconds). However, we have previously argued that the information which is presumably needed to trigger anticipatory responses with the appropriate timing relies on continuous sensory inputs providing instantaneous estimates of the time-to-contact (Lacquaniti and Maioli, 1989).

\section{Short-latency reflex responses}

These responses consisted of a pattern of strict coactivation of the antagonist muscles at the elbow and wrist joints that did not differ with eyes open or closed. This pattern, as well as its putative neural substrates, has been discussed in the companion paper. The present experiments provided further evidence in favor of the contention that the reflex coactivation is centrally preset. In fact, when the auditory cue for ball release was unexpectedly suppressed in the absence of vision, the mean amplitude of the short-latency EMG responses was significantly smaller than when the subjects were alerted, as was the coactivation ratio between antagonist muscles (Fig. 5). The considerable degradation in catching performance that occurred in such cases further emphasizes the functional significance of presetting the reflex coactivation vis-à-vis the need for limb stabilization. Note that, in contrast to the responses of wrist and elbow muscles, the short-latency responses of the FPL were much larger with eyes closed than with eyes open (Fig. 1, C, D).

\section{Medium-latency responses}

In contrast to the short-latency responses to impact, mediumlatency responses tended to be reciprocally organized in the antagonist muscles at both the elbow and wrist joints. They share this fundamental principle of organization with the EMG responses traditionally described in load perturbation experi- 
ments (see Dufresne et al., 1978; Lacquaniti and Soechting, 1986a). An unexpected result was that their amplitude was dramatically affected by the visual condition tested, being much larger in the absence of vision than in its presence. Three questions need therefore to be addressed: (1) What might be the adequate stimulus for the elicitation of such responses? (2) What might be their neural substrates? (3) How can the visual conditions influcnce their amplitude? Different hypotheses will be herein considered.

First, the contact forces resulting from the impact of the ball on the hand might be encoded in the output of cutaneous receptors and represent the adequate stimulus for the mediumlatency EMG responses. While this hypothesis may apply to the responses of FPL (Cole and Abbs, 1987; Johansson and Westling, 1987), it is unlikely for the responses of wrist and elbow muscles. In fact, the distribution of the EMG responses elicited by means of electrical or mechanical stimulation of skin afferents from the hand is essentially limited to hand muscles; moreover, the responses in antagonist muscles are nonreciprocal (Caccia et al., 1973).

The adequate stimulus for the medium-latency responses of wrist and elbow muscles is presumably represented by the changes in kinematic variables (e.g., angular velocity) or dynamic variables (torque) at the elbow and wrist joints following the impact. Indeed, when a torque motor perturbation is applied to a single limb segment and motion of the other segments is restricted, the direction and amplitude of the medium-latency responses are strongly correlated with those of the preceding changes in the angular velocity (Dufresne et al., 1979; Gottlieb and Agarwal, 1979; Jaeger et al., 1982). Negative feedback of the rate of change of muscle length provided by muscle spindles (Matthews and Stein, 1969; Poppele and Bowman, 1970) has been implicated to account for such responses. More recently, the EMG responses to load perturbations of the upper limb have been investigated under conditions in which angular motion at the shoulder and elbow joints is permitted (Lacquaniti and Soechting, 1986a, b). Under such conditions, the average amplitude of the "early" responses (latency of about $40 \mathrm{msec}$ ) is best correlated with the average changes in angular velocity, while that of the "late" responses (latency of about $80 \mathrm{msec}$ ) is best correlated with the average changes in net torque resulting from the perturbation (Soechting and Lacquaniti, 1988).

In the present experiments, since the perturbation acted distally to the wrist joint and about orthogonally to both the hand and the forearm, the main changes in angular velocity at the elbow and wrist resulting from the impact were in the same direction (extension) as the changes in torque. Thus, we cannot dissociate the effect of kinematics from that of dynamics; the reciprocal EMG responses we observed appear compatible with feedback of either angular velocity or torque (a net change of velocity and torque towards extension leading to activation of flexor muscles and disfacilitation of extensor muscles). However, the large difference in amplitude of such responses according to the visual conditions cannot be explained by considering only elbow and wrist kinematics or dynamics, whose amplitude and time course over the first 50-100 msec after impact varied only to a limited extent in the 2 conditions (see Fig. 1).

In contrast to elbow and wrist kinematics, $\mathrm{MCP}$ kinematics differed markedly according to the visual conditions present during the first $80 \mathrm{msec}$ after impact (see Fig. 4). While monotonic flexion of the fingers occurred with vision, triphasic changes (flexion-extension-flexion) occurred without vision. One might therefore hypothesize that finger extension leads to excitation of $\alpha$-motoneurons of flexor muscles at the wrist and elbow joints and inhibition of those of extensor muscles. For instance, this might be due to the heteronymous connections from Ia and II spindle afferents from finger muscles (Clough et al., 1968).

Nonautogenetic feedback has previously been implicated to account for the EMG responses of monoarticular elbow muscles evoked by load perturbations applied to the upper limb free to move (Lacquaniti and Soechting, 1986b). Such responses depend, in fact, on elbow and shoulder angular motion. However, kinematic studies of intentional movements of the upper limb (reaching and drawing) have demonstrated that the control of distal joints (wrist and fingers) is separate from that of proximal joints (shoulder and elbow): while shoulder and elbow angular motions are tightly coupled, motion at the distal joints is largely uncoupled from the motion at the proximal joints (Lacquaniti and Soechting, 1982; Lacquaniti et al., 1987). Thus, it is unlikely that the reflex control of elbow and wrist muscles during catching can be influenced by finger motion to the extent required to explain the large difference in the magnitude of the responses in the 2 visual conditions.

In sum, it appears that the mechanical factors per se can hardly account for such a difference. Instead, one has to assume that the gain of the medium-latency responses is differently preset by central processes, being larger in the absence of vision than in its presence. Task-dependent variations of the gain of medium-latency EMG responses evoked by load perturbations are known; for instance, the gain is larger when subjects are instructed to resist the perturbation than when they do not resist (Hammond, 1956; Marsden et al., 1976; Dufresne et al., 1979; Jaeger et al., 1982). In a similar vein, the increment of the amplitude of medium-latency responses during catching without vision would depend on the intended goal of stabilizing limb posture in the absence of anticipatory muscle stiffening. Visual inputs might influence on line the excitability of $\alpha$-motoneurons (Anderson et al., 1972). Such an influence has been postulated to account for the depression of medium-latency EMG responses of ankle extensors during free-fall with stabilized vision (Vidal et al., 1979). Note, however, that the nature of the visuospinal influences involved in the 2 cases would be clearly different (facilitation of EMG responses by normal visual inputs during postural perturbations, as opposed to the depression postulated in the present case).

\section{Long-latency responses}

The latency of these responses makes them comparable to the "late" responses that have been described in elbow and shoulder muscles during multijoint limb perturbations (Lacquaniti and Soechting, 1986b; Soechting and Lacquaniti, 1988). These "late" responses have been shown to depend on a negative feedback of net joint torques. However, it should be noted that, when the perturbation is predictable (as in the present experiments), rcaction-time contributions cannot be ruled out. In fact, the variability in the pattern and amplitude of the responses we observed is more typical of the latter than of reflex responses (see Houk, 1978).

Also these EMG responses were gated as a function of the visual condition, being appreciable only without vision. The different preset of these responses according to the task is in agreement with the well-known dependence of the amplitude of long-latency responses on central set (Houk, 1978; Jaeger et 
al., 1982). Direct influences of visual inputs onto long-latency responses of ankle extensors to anteroposterior body sway have been also demonstrated (Nashner and Berthoz, 1978).

\section{Adaptive calibration of the responses}

The present study shed no light on the neural mechanisms underlying the presetting of the muscle responses elicited by the ball's impact. Multiple processes are presumably at play in modifying the parameters (gain and dynamics) of responses having different latencies and neural substrates (see Terzuolo et al., 1981). Whatever the specific nature of these processes, 2 points can be made about their operational properties. First, the control of response parameters is likely to be time-varying. Thus, as demonstrated when the second tone was suppressed (Fig. 5), the changes in the reflex excitability of $\alpha$-motoneurons, which are required for effective catching, were not set up from the beginning of a trial but were contingent upon the arrival of sensory cues on the timing of the fall. Considering previous studies on the time-varying properties of myotatic responses during simple tasks (Soechting et al., 1981), one might speculate that the response parameters change with a time course that is related to the expected time of impact. This hypothesis is amenable to experimental verification.

The second point concerns the adaptive changes of the responses in the course of an experiment. The general pattern of the EMG responses was switched from that characteristic of catching with vision (consisting of anticipatory activity and brief reflex responses) to that characteristic of catching without vision (no anticipatory activity and prolonged responses to impact) with the very first trial of each experiment. However, the responses produced without vision were presumably calibrated adaptively. In fact, the limb oscillations induced by the impact were significantly larger in the first trial than in the following trials (Figs. 6, 7). One has therefore to assume that changes appropriate to damping limb oscillations took place in the amplitude and time course of the EMG responses after the first trial (although the EMG parameters we considered did not reveal consistent trends).

We suggest the following as one possible scheme of organization of this adaptive system. An internal model of the dynamic interaction that is expected to occur between the falling ball and the limb is constructed based on a priori knowledge. The response of the model to the actual disturbance is compared with the mechanical response of the limb to produce an error signal. This error is subsequently used (1) to calibrate the parameters of the reflex controller and (2) to update the model. This scheme belongs to the class of "model reference adaptive systems" (see Åström, 1983). The present data indicate that, initially, the model does not accurately predict the desired performance, possibly because of an erroneous estimate of ball momentum. This estimate, in fact, can be based only on a prior knowledge of the height of the drop and the mass of the ball, without the supplementary information on ball velocity which is provided by visual feedback (Lacquaniti and Maioli, 1989). Kinesthetic and cutaneous information obtained during the first trial can be used to correct the estimate and achieve the desired performance in subsequent trials.

\section{References}

Abbs, J. H., and K. J. Cole (1987) Neural mechanisms of motor equivalence and goal achievement. In Higher Brain Functions, S. P. Wise, ed., pp. 15-43, Wiley, New York.
Anderson, M. E., M. Yoshida, and V. J. Wilson (1972) Tectal and segmental influences on cat forelimb and hindlimb motoneurons. J. Neurophysiol. 35: 462-470.

Åström, K. J. (1983) Theory and applications of adaptive control. A survey. Automatica 19: 471-486.

Berthoz, A. (1988) The role of gaze in compensation of vestibular disfunction. Prog. Brain Res. (in press).

Berthoz, A., M. Lacour, J. F. Soechting, and P. P. Vidal (1979) The role of vision in the control of posture during linear motion. Prog. Brain Res. 50: 197-209.

Caccia, M. R., A. J. McComas, A. R. M. Upton, and T. Blogg (1973) Cutaneous reflexes in small muscles of the hand. J. Neurol. Neurosurg. Psychiatry 36: 960-977.

Clough, J. F. M., D. Kernell, and C. G. Phillips (1968) The distribution of monosynaptic excitation from the pyramidal tract and from primary spindle afferents to motoneurons of the baboon's hand and forearm. J. Physiol. (Lond.) 198: 145-166.

Cole, K. J., and J. H. Abbs (1987) Kinematic and electromyographic responses to perturbation of a rapid grasp. J. Neurophysiol. 57: 14981510.

Dichgans, J., E. Bizzi, P. Morasso, and V. Tagliasco (1973) Mechanisms underlying recovery of the eye-head coordination following bilateral labyrinthectomy in monkeys. Exp. Brain Res. 18: 548-562.

Dufresne, J. R., J. F. Soechting, and C. Terzuolo (1978) Electromyographic response to pseudo-random torque disturbances of human forearm position. Neuroscience 3: 1213-1226.

Dufresne, J. R., J. F. Soechting, and C. Terzuolo (1979) Reflex motor output to torque pulses in man: Identification of short- and longlatency loops with individual fccdback parametcrs. Neuroscience 4: 1493-1500.

Dyhre-Poulsen, P., and A. M. Laursen (1984) Programmed electromyographic activity and negative incremental muscle stiffness in monkeys jumping downward. J. Physiol. (Lond.) 350: 121-136.

Gottlieb, G. L., and G. C. Agarwal (1979) Response to sudden torques about ankle in man: Myotatic reflex. J. Neurophysiol. 42: 91-106.

Greenwood, R. G., and A. Hopkins (1976) Muscle responses during sudden falls in man. J. Physiol. (Lond.) 254: 507-518.

Hammond, P. H. (1956) The influence of prior instruction to the subject on an apparently involuntary neuromuscular response. $J$. Physiol. (I ond.) 132: 17-18.

Houk, J. C. (1978) Participation of reflex mechanisms and reactiontime processes in the compensatory adjustments to mechanical disturbances. Prog. Clin. Neurophysiol. 4: 193-215.

Jaeger, R. J., G. L. Gottlieb, and G. C. Agarwal (1982) Myoelectric responses at flexors and extensors of human wrist to step torque perturbations. J. Neurophysiol. 48: 388-402.

Johansson, R. S., and G. Westling (1987) Signals in tactile afferents from the fingers eliciting adaptive motor responses during precision grip. Exp. Brain Res. 66: 141-154.

Lacour, M., P. P. Vidal, and C. Xerri (1981) Visual influences on vestibulospinal reflexes during vertical linear motion in normal and hemilabyrinthectomized monkeys. Exp. Brain Res. 43: 383-394.

Lacquaniti, F., and C. Maioli (1989) The role of preparation in tuning anticipatory and reflex responses during catching. J. Neurosci. 9: 134148.

Lacquaniti, F., and J. F. Soechting (1982) Coordination of arm and wrist motion during a reaching task. J. Neurosci. 2: 399-408.

Lacquaniti, F., and J. F. Soechting (1986a) EMG responses to load perturbations of the upper limb: Effect of dynamic coupling between shoulder and elbow motion. Exp. Brain Res. 61: 482-496.

Lacquaniti, F., and J. F. Soechting (1986b) Responses of mono- and bi-articular muscles to load perturbations of the human arm. Exp. Brain Res. 65: 135-144.

Lacquaniti, F., G. Ferrigno, A. Pedotti, J. F. Soechting, and C. Terzuolo (1987) Changes in spatial scale in drawing and handwriting: Kinematic contributions by proximal and distal joints. J. Neurosci. 7 : 819-828.

Maioli, C. (1988) Optokinetic and vestibular oculomotor responses and their interaction following hemilabyrinthectomy in the cat. In Post-Lesion Neural Plasticity, H. Flohr, ed., pp. 381-391, SpringerVerlag, Berlin.

Marsden, C. D., P. A. Merton, and H. B. Morton (1976) Stretch reflex and servo action in a variety of human muscles. J. Physiol. (Lond.) 259: 531-560.

Matthews, P. B. C., and R. B. Stein (1969) The sensitivity of muscle 
spindle afferents to small sinusoidal changes in length. J. Physiol. (Lond.) 200: 723-743.

McKinley, P. A., and J. L. Smith (1983) Visual and vestibular contributions to prelanding EMG during jump-down in cats. Exp. Brain Res. 52: 439-448.

Melvill Jones, G., D. Guitton, and A. Berthoz (1988) Changing patterns of eye-head coordination during $6 \mathrm{hrs}$ of optically reversed vision. Exp. Brain Res. 69: 531-544.

Nashner, L. M., and A. Berthoz (1978) Visual contribution to rapid motor responses during postural control. Brain Res. 150:403-407.

Nashner, L. M., F. O. Black, and C. Wall (1982) Adaptation to altered support and visual conditions during stance: Patients with vestibular deficits. J. Neurosci. 2: 536-544.

Poppele, R. E., and R. J. Bowman (1970) Quantitative description of linear behavior of mammalian muscle spindles. J. Neurophysiol. 33: 59-72.

Putkonen, P. T. S., J. H. Courjon, and M. Jeannerod (1977) Compensation of postural effects of hemilabyrinthectomy in the cat. A sensory substitution process? Exp. Brain Res. 28: 249-257.

Sharp, R. H., and H. T. A. Whiting (1974) Exposure and occluded duration effects in a ball catching skill. J. Motor Behav. 6: 139-147.
Soechting, J. F., and F. Lacquaniti (1988) Quantitative evaluation of the electromyographic responses to multi-directional load perturbations of the human arm. J. Neurophysiol. 59: 1296-1313.

Soechting, J. F., J. R. Dufresne, and F. Lacquaniti (1981) Time-varying properties of myotatic response in man during some simple motor tasks. J. Neurophysiol. 46: 1226-1243.

Talbott, R. E., and J. M. Brookhart (1980) A predictive model study of the visual contribution to canine postural control. Am. J. Physiol. 239: R80-R92.

Terzuolo, C., J. F. Soechting, and J. R. Dufresne (1981) Operational characteristics of reflex responses to changes in muscle length during different motor tasks and their functional utility. In Brain Mechanisms and Perceptual Awareness, O. Pompeiano and C. Ajmone Marsan, eds., pp. 183-210, Raven, New York.

Vidal, P. P., M. Lacour, and A. Berthoz (1979) Contribution of vision to muscle responses in monkey during free-fall: Visual stabilization decreases vestibular-dependent responses. Exp. Brain Res. 37: 241252. 